\title{
两种不同训练时程方法对海马损伤大鼠水迷宫成绩的影响
}

\author{
边 慧 ${ }^{1}$, 方 松 ${ }^{1,2}$, 缪应达 ${ }^{3}$, 余化霖 ${ }^{1, *}$ \\ (1. 昆明医学院, 云南 昆明 650032; 2. 南昌大学 第一附属医院医务科, 江西 南昌 330006; \\ 3. 中国人民银行 昆明市中心支行, 云南 昆明 650223)
}

\begin{abstract}
摘要: 为探讨训练时程对大鼠水迷宫成绩的影响, 采用直流电解法建立双侧背侧海马损伤大鼠模型, 并通过 5 $\mathrm{d}(5 \mathrm{~d}$ training)和 $7 \mathrm{~d}$ 训练 (7d training)两种不同时程法检测海马损伤大鼠的空间学习记忆能力。结果表明, 两种训 练法均反映大鼠空间学习能力减退, 而在检测空间记忆障碍时则略有不同。与 $5 \mathrm{~d}$ 训练法相比, $7 \mathrm{~d}$ 训练法不仅显 示大鼠穿台次数下降, 且其在目标象限中的停留时间百分比亦下降。
\end{abstract}

关键词: 水迷宫; 空间学习记忆; 海马损伤

中图分类号：R338; Q42 文献标志码: A 文章编号: 0254-5853-(2012)02-0237-04

\section{Influences of two different training paradigms on spatial learning and memory performance of hippocampal injured rats}

\author{
BIAN Hui ${ }^{1}$, FANG Song ${ }^{1,2}$, MIAO Ying-Da ${ }^{3}$, YU Hua-Lin ${ }^{1, *}$ \\ (1. Kunming Medical College, Kunming 650032, China ; 2. Medical Department, First Affiliated Hospital of Jiangxi Medical University, Nanchang 330006 , \\ China ; 3. Kunming Branch, People's Bank of China, Kunming 650223, China)
}

\begin{abstract}
In this study, the $5 \mathrm{~d}$ and $7 \mathrm{~d}$ training paradigms were adopted to investigate the influences of different training procedures on the performance of spatial learning and memory of the hippocampal injured rats. The results showed that during the hidden-platform acquisition training, similar spatial learning dysfunctions were indicated in those two training paradigms. Whereas, when the spatial memories have been evaluated, compared with the $5 \mathrm{~d}$ training groups, the rats under $7 \mathrm{~d}$ training procedure not only crossed the platform location less, but also preferred to spend less time in the target quadrant.
\end{abstract}

Key words: Morris water maze; Spatial learning and memory; Hippocampal injury

Morris 水迷宫(Morris water maze, MWM)实验 由英国心理学家 Morris 于 1981 年建立, 并随后进 行了不断的完善及改进, 最初用于研究大鼠的空间 学习记忆功能(Morris, 1981，1984)。目前 MWM 已 成为研究实验动物空间学习和记忆能力最常用的 实验方法之一, 广泛应用于神经科学、药学等领域 (D’Hooge \& De Deyn, 2001)。

MWM 实验是依赖于海马的空间学习记忆任务, 而该任务的完成以海马结构、功能的完整性为前提 (D’Hooge \& De Deyn, 2001)。当不同原因导致海马 结构或功能不完整时会损害动物的空间学习记忆
能力, 进而影响该动物的 MWM 成绩(Morris et al, 1982; Morris, 1984)。

自 MWM 建立以来，已有诸多研究者根据不同 实验目的对经典 MWM 进行调整, 建立了多种 MWM 实验方案(Vorhees \& Williams, 2006)。不同研 究者在水迷宫具体操作方面大致相同, 但又稍有区 别。例如，隐藏平台实验由多个训练日构成，其目 的是使动物在连续多日的训练中学会登上隐藏平 台的实验规则, 并理解如何得到救援 (Hodges, 1996)。但在此过程中每天具体的训练次数以及总的 训练时程并没有统一要求, 每天的训练次数从 2

收稿日期: 2011-08-09; 接受日期: 2011-11-20

基金项目: “973”计划 (2007CB947703)

*通信作者(Corresponding author), E-mail: yuh1308@126.com

第一作者简介: 边慧, 昆明医学院 08 级博士, E-mail: bh_032001@yahoo.com.cn 
10 次不等, 训练时程多为 $6 \sim 15$ 个训练日。那么, 不 同的训练时程能否对 MWM 成绩产生不同的影响, 尤其是该影响是否依赖于海马的完整性, 目前尚无 明确答案。为此, 本研究拟采用 MWM 来检测直流 电解法建立的双侧背侧海马损伤大鼠的空间学习 记忆能力, 观察 $5 \mathrm{~d} 、 7 \mathrm{~d}$ 两种不同训练时程的方法 是否影响其 MWM 成绩，从而寻找一种更适合本实 验模型的训练方法, 为后续实验奠定基础。

\section{1 材料和方法}

\section{1 实验动物}

成年雄性 Sprague-Dawley 大鼠购于昆明医学 院实验动物中心, 购买时体重 $180 \sim 200 \mathrm{~g}$, 许可证 编号为 SCXK(滇)2005-0008。实验开始前, 所有实 验用鼠在本实验室适应至少一周。大鼠饲养在动物 笼内, 自由进水取食, $12 \mathrm{~h}$ 明、暗交替(光照时间: 07: 00-19: 00), 室温 $(23 \pm 2){ }^{\circ} \mathrm{C}$ 。操作符合动物实 验管理条例。

\section{2 动物模型建立及分组}

大鼠设置为 $5 \mathrm{~d}$ 训练组: 对照 (control)8 只、 海马损伤 (lesion) 11 只; $7 \mathrm{~d}$ 训练组：对照 (control)12 只、海马损伤 (lesion)11 只。

动物模型建立: 大鼠腹腔注射硫酸阿托品 $(0.3 \mathrm{~mL} /$ 只, 天津金耀氨基酸有限公司)及戊巴比妥 钠 $(45 \mathrm{mg} / \mathrm{kg}$ 体重, Sigma)致麻醉后, 将其固定在脑 立体定位仪上(深圳瑞沃德生命科技有限公司)。依 次切开头皮、肌肉以暴露频骨, 在海马上方颖骨钻 孔 4 个, 孔径约为 $1 \mathrm{~mm}$ 。将仅尖端暴露 $1 \mathrm{~mm}$ 的涂 有绝缘漆的不锈钢针负针 (直径为 $0.3 \mathrm{~mm}$, 苏州医 疗用品厂)垂直插入大鼠背侧海马。根据大鼠脑立体 定位图谱 (Paxinos \& Watson, 1998)确定大鼠背侧海 马坐标: 前囟后 $4 \mathrm{~mm}$, 中线左/右旁 $2 \mathrm{~mm}$ 及 $3.4 \mathrm{~mm}$, 乑骨下 $4 \mathrm{~mm}$ 。打开直流电损毁仪进行直流电解, 参数为 $2 \mathrm{~mA}, 60 \mathrm{~s}$ 。手术结束后缝合头皮, 局部涂擦 红霉素软膏, 同时连续 $3 \mathrm{~d}$ 肌肉注射青霉素钠 $4 \times$ $10^{6} \mathrm{U}$ 预防感染。手术后 4 周进行水迷宫检测。

\section{3 水迷宫检测}

本实验所用水迷宫为一直径 $180 \mathrm{~cm}$ 、高 $50 \mathrm{~cm}$ 的圆形水池, 池内水深 $31 \mathrm{~cm}$ (适应阶段为 $29 \mathrm{~cm}$ ), 水温维持在 $(21 \pm 1){ }^{\circ} \mathrm{C}$ 。黑色遮光帘将水池围起以减 少其他因素的干扰, 遮光布帘上贴有不同形状颜色 图形作为标记。实验间内光线恒定, 水面漂浮覆盖 褐色塑料颗粒, 使大鼠看不见平台和池底。水迷宫
被等分为第 I 、II、III、IV象限, 参照 Vorhees \& Williams (2006)的方法取西北、北、东、东南等 4 个入水点, 平台置放于第 II 象限(目标象限)正中。水 迷宫实验由熟悉适应、隐藏平台及空间探索三部分 组成。第一天为熟悉适应过程，逃逸平台暴露且高 出水面 $1 \mathrm{~cm}$, 将大鼠面壁放入水迷宫自由游泳 120 $\mathrm{s}$ 适应环境, 记录大鼠寻求并登上平台所需的时间, 即逃避潜伏期(latency); 若 $120 \mathrm{~s}$ 后大鼠未找到平台, 则将其引导至平台并停留 $60 \mathrm{~s}$; 共进行 4 次训练。 隐藏平台实验历时 $5 \mathrm{~d}$ (或 $7 \mathrm{~d}$ ), 逃逸平台隐藏于水 下 $1 \mathrm{~cm}$ 。每天每只大鼠接受 4 次训练, 记录方式同 适应阶段, 具体训练流程参照 Vorhees \& Williams (2006)。空间探索实验在最后一次隐藏平台实验结 束后 $24 \mathrm{~h}$ 开始,撤除平台, 将大鼠从第III象限(目标 象限的对面象限)中点放入水中, 记录 $120 \mathrm{~s}$ 内大鼠 在 4 个象限的游泳时间百分比及穿过原平台所在位 置的次数。迷宫上方安装连接显示系统的摄像头, 大鼠的运动轨迹的记录和分析采用中国科学院昆 明动物研究所研制开发的软件完成。

水迷宫检测前 1 周进行大鼠的运动功能相关检 测(balance beam 和 prehensile-traction)以评价其运 动功能。结果显示, 损伤组与对照组大鼠运动功能评 分没有统计学差异, 提示损伤组大鼠无运动障碍。

\section{4 组织学检查}

实验结束后对电极损伤区域进行组织学检查, 分别用生理盐水及 $4 \%$ 多聚甲醛经左心室灌流后取 脑。多聚甲醛固定, 20\%、30\%蔗糖溶液梯度脱水后， 做冰冻冠状切片, 片厚 $20 \mu \mathrm{m}$, 然后进行 $\mathrm{HE}$ 染色观 察海马损伤情况。若定位不在背侧海马的动物弃之 不用。

\section{5 统计学分析}

数据以 mean $\pm S E$ 表示, 水迷宫的逃避潜伏期采 用重复测量方差分析; 空间探索实验中的各象限游 泳时间百分比采用单因素方差分析，组间比较及空 间探索实验中的穿台次数采用非配对 $t$-检验分析。 所有统计计算由 SPSS13.0 软件完成, $P \leqslant 0.05$ 视为 具有统计学差异。

\section{2 结 果}

\section{1 组织学检查}

切片经 HE 染色显示, 模型组大鼠海马组织结 构不完整, 椎体细胞层破坏, 大量细胞缺失, 排列紊 乱疏松, 损伤区域主要位于背侧海马及其周围(图 1)。 


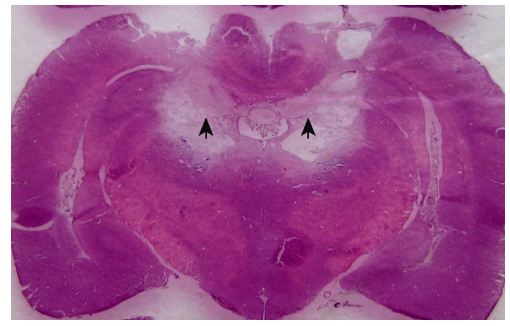

图 1 直流电解损伤大鼠双侧背海马(HE 染色法)

Fig.1 Bilateral dorsal electrolytic injured hippocampus (Hematoxylin-Eosin staining)

\section{2 水迷宫空间学习能力比较}

将大鼠每天 4 次的逃避潜伏期取平均值得到每 天的水迷宫平均逃避潜伏期。重复测量方差分析显 示, $5 \mathrm{~d}$ 训练组对照、海马损伤大鼠的平均逃避潜伏 期总体比较具显著性差异 $\left[F_{(1,17)}=4.98, P<0.05\right]$ 。与 对照组相比, 海马损伤大鼠的逃避潜伏期明显延长 [非配对 $t$-检验: $\quad t=-2.74, P<0.05(\mathrm{~d} 4) ; t=-2.40, P$ $<0.05$ (d5); $\mathrm{t}=-4.30, P<0.01$ (d6)](图 2)。 $7 \mathrm{~d}$ 训练 组对照、海马损伤大鼠的平均逃避潜伏期总体比较 有显著性差异 $\left[F_{(1,21)}=26.10, P<0.01\right]$ 。与对照相比, 海马损伤大鼠的逃避潜伏期明显延长 [非配对 $t$-检 验: $t=-3.88, P<0.01(\mathrm{~d} 2) ; t=-4.47, P<0.01(\mathrm{~d} 3)$; $t=-3.79, P<0.01(\mathrm{~d} 4) ; t=-4.43, P<0.01(\mathrm{~d} 5) ; t=-4.69$, $P<0.01(\mathrm{~d} 6) ; t=-3.59, \quad P<0.01(\mathrm{~d} 7) ; t=-3.18, \quad P<$ 0.01(d8)]（图 3)。

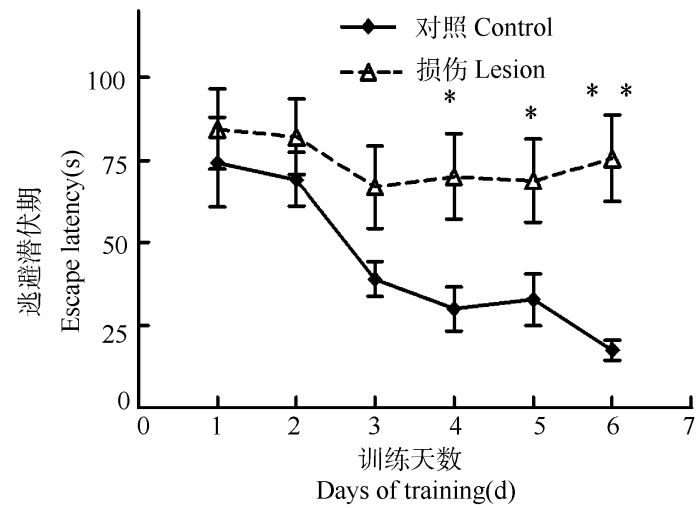

图 $25 \mathrm{~d}$ 训练组平均逃避潜伏期

Fig. 2 Average latency of the $5 \mathrm{~d}$ training group $*: P<0.05 ; * *: P<0.01$

\section{3 水迷宫空间记忆能力比较}

空间探索实验结果显示 $5 \mathrm{~d}$ 训练组、 $7 \mathrm{~d}$ 训练组 海马损伤大鼠穿台次数明显减少, 与对照组相比, 有显著性差异[非配对 $t$-检验：ST $t=3.10, P<0.01$; LT $t=-5.92, P<0.01$ ] (图 4)。 $5 \mathrm{~d}$ 训练组海马损伤大 鼠在目标象限游泳时间百分比高于对面象限 [LSD: target quadrant $v s$ opposite quadrant $P<0.05$ ]; $7 \mathrm{~d}$ 训练
组海马损伤大鼠在目标象限游泳时间百分比下降, 与对面象限游泳时间百分相比，无显著性差异[LSD: target quadrant $v s$ opposite quadrant $P>0.05]$ (图 5)。

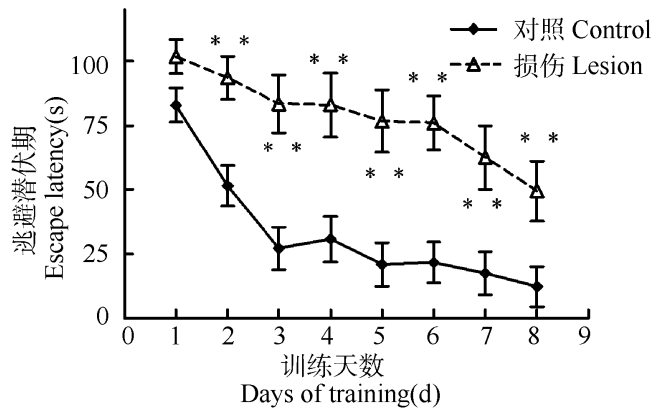

图 $37 \mathrm{~d}$ 训练组平均逃避潜伏期

Fig.3 Average latency of the $7 \mathrm{~d}$ training group $* *: P<0.01$

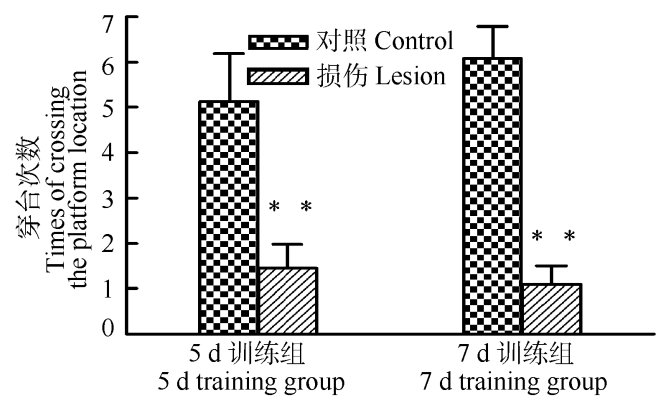

图 $45 \mathrm{~d}$ 训练组、 $7 \mathrm{~d}$ 训练组穿台次数

Fig. 4 Times of crossing the platform location of the $5 \mathrm{~d}$ and $7 \mathrm{~d}$ training group

**: $P<0.01$

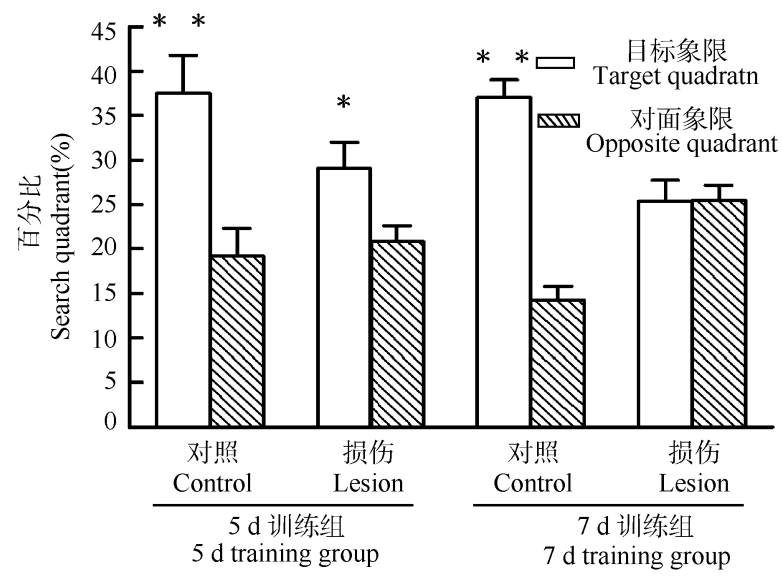

图 $55 \mathrm{~d} 、 7 \mathrm{~d}$ 训练组在目标象限及对面象限的游泳时间百分比 Fig.5 The swimming time percentages of $5 \mathrm{~d}$ and $7 \mathrm{~d}$ training groups in the target quadrant vs opposite quadrant $*: P<0.05 ; * *: P<0.01$

\section{3 讨 论}

本研究采用两种不同时程的训练方法来探讨 训练时程是否会对双侧背侧海马损伤大鼠的水迷 
宫成绩产生影响。结果发现, 在隐藏平台实验中, 两种训练法的海马损伤大鼠均表现为逃避潜伏期 明显延长, 提示大鼠的空间学习能力下降。该结果 与前人报道一致(Wood et al, 1993;Rapp \& Gallagher, 1996)。在空间探索实验中, $5 \mathrm{~d}$ 训练组海马损伤大鼠 不能对平台进行精确定位, 穿台次数明显下降, 但 对目标象限仍有一定偏好; 当把训练时程延长 $2 \mathrm{~d}$ 后, 模型大鼠在空间探索实验中不表现明显象限偏好, 提示大鼠空间记忆能力下降, 即较长时程的训练可 以更彻底地反映大鼠背侧海马损伤的影响。

逃避潜伏期是隐藏平台实验中的一个相对稳 定的评价指标。以往的研究发现, 不同实验室之间 MWM 的实验设备、训练方法、动物种类及周围环 境等或多或少存在差异, 但逃避潜伏期受这些因素 的影响较小, 检测结果差别不大(Crabbe et al, 1999), 本实验结果也验证了这一点。当改变训练时程时, 海马损伤大鼠均表现明显的空间学习障碍, 说明两 种训练方法均能检测出动物空间学习能力的下降。 空间探索实验是在一系列重复的隐藏平台实验的 基础上进行的, Morris 以成年正常雄性 Lister 大鼠为 实验动物, 最初采取的是第一、二天各 8 次, 第三 天 4 次, 共训练 20 次后进行空间探索实验的做法, 结果显示空间探索实验中大鼠在目标象限停留时 间明显高于其他象限(Morris, 1981); 在随后的实验 中, 采用每天 4 次, 共训练 28 次后进行空间记忆功 能检测的方法, 结果发现海马背侧和腹侧均损伤的 大鼠无明显的象限偏好, 而海马上方皮层损伤及空 白对照组大鼠, 则均表现出明显的象限偏好, 且在 目标象限停留的时间高于其他象限(Morris et al,

\section{参考文献:}

Crabbe JC, Wahlsten D, Dudek BC. 1999. Genetics of mouse behavior: interactions with laboratory environment[J]. Science, 284(5420): 1670-1672.

D’Hooge R, De Deyn PP. 2001. Applications of the Morris water maze in the study of learning and memory[J]. Brain Res Rev, 36(1): 60-90.

Hodges H. 1996. Maze procedures: the radial-arm and water maze compared[J]. Cogn Brain Res, 3(3-4): 167-181.

Morris R. 1984. Developments of a water-maze procedure for studying spatial learning in the rat[J]. $J$ Neurosci Meth, 11(1): 47-60.

Morris RGM. 1981. Spatial localization does not require the presence of local cues[J]. Learn Motiv, 12(2): 239-260.

Morris RGM, Garrud P, Rawlins JNP, O’Keefe J. 1982. Place navigation impaired in rats with hippocampal lesions[J]. Nature, 297(868): 681-683.

Moser E, Moser MB, Andersen P. 1993. Spatial learning impairment parallels the magnitude of dorsal hippocampal lesions, but is hardly present following ventral lesions[J]. J Neurosci, 13(9): 3916-3925.

Moser MB, Moser EI, Forrest E, Andersen P, Morris RG. 1995. Spatial
1982)。Moser et al (1995)使用鹅膏草氨酸损伤大鼠 双侧背海马, 然后每天 8 次, 共训练 32 次后检测大 鼠的空间记忆功能, 发现海马损伤大鼠无明显象限 偏好。然而, 由于每天训练 8 次工作量很大, 故现 在多采用每天训练 4 次的方法, 但不同研究者仍有 其各异的训练法(Moser et al, 1993; Riedel et al, 1999)。我们在前期进行空间探索实验时发现, 5 d 训练组海马损伤大鼠不能对平台进行精确定位, 穿 台次数明显下降, 但对目标象限仍有一定的偏好。 考虑到海马损伤大鼠只有经过 “足够” 的训练才可 以达到提取记忆的目的, 我们将训练时程延长后发 现大鼠穿台次数明显下降且无明显象限偏好，提示 大鼠的空间记忆能力明显下降。两种时程的方法产 生差异的原因可能是由于海马损伤大鼠的海马结 构功能异常。对照组大鼠, 经过短期的训练足以达 到稳定的空间学习记忆状态, 而海马损伤大鼠则需 要延长训练时程才可以达到一个相对稳定的状态。

综上所述, 本实验结果表明两种不同时程的训 练方法均能反映海马损伤大鼠的空间学习能力, 但 在检测空间记忆方面略有不同。当用于检测动物的 空间学习能力时倾向于选择 $5 \mathrm{~d}$ 训练法, 而检测空 间记忆能力时则可选择 $7 \mathrm{~d}$ 训练法。水迷宫实验作 为测试动物空间学习记忆能力的经典方法, 值得进 一步研究、完善。

致谢: 感谢中科院昆明动物所马原野研究员、 胡新天研究员给予的帮助, 感谢王正波博士在实 验操作方面给予的帮助, 感谢于程洋同学在水迷 宫实验中给予的帮助。

learning with a minislab in the dorsal hippocampus[J]. Proc Natl Acad Sci USA, 92(21): 9697-9701.

Paxinos G, Watson C. 1998. The Rat Brain in Stereotaxic Coordinates[M]. 4th ed. San Diego: Academic Press.

Rapp PR, Gallagher M. 1996. Preserved neuron number in the hippocampus of aged rats with spatial learning deficits[J]. Proc Natl Acad Sci USA, 93(18): 9926-9930.

Riedel G, Micheau J, Lam AGM, Roloff EVL, Martin SJ, Bridge H, De Hoz L, Poeschel B, McCulloch J, Morris RGM. 1999. Reversible neural inactivation reveals hippocampal participation in several memory processes[J]. Nat Neurosci, 2(10): 898-905.

Vorhees CV, Williams MT. 2006. Morris water maze: procedures for assessing spatial and related forms of learning and memory[J]. Nat Protoc, 1(2): 848-858.

Wood ER, Mumby DG, Pinel JP, Phillips AG. 1993. Impaired object recognition memory in rats following ischemia-induced damage to the hippocampus[J]. Behav Neurosci, 107(1): 51-62. 